\title{
Impact of operation on the geometric parameters of the track in ballastless track transition area
}

\author{
Libor Ižvolt ${ }^{1}$, Michal Šmalo ${ }^{1 *}$ and Janka Šestáková ${ }^{1}$ \\ ${ }^{1}$ University of Žilina, Faculty of Civil Engineering, Department of Railway Engineering and Track \\ Management, Univerzitná 8215/1, 01026 Žilina, Slovakia
}

\begin{abstract}
The paper deals with the methodology and results of diagnostics in test sections near the tunnel portals of newly built tunnel Turecký vrch on modernized line Nové Mesto nad Váhom - Púchov. Based on the diagnostics results, transition areas are problematic sections of the construction. Transition areas were subjected to detailed analysis and based on trends of the development of geometric quantities are determined trends which predict time of needed maintenance. In order to optimize existing transition areas the design of transition in ANSYS software was modelled and problematic spots of construction were defined.
\end{abstract}

\section{Introduction}

The increasing mobility of population and the competition among various transportation systems pose new challenges in the field of railway transport. An essential prerequisite for competitiveness of railway transport is reliable operation of railway tracks, which can be understood as a safe and stable movement of rail vehicles along the track.

The development and verification of various technical solutions of ballastless track demonstrate the efforts to optimize the railway superstructure. An upgraded superstructure would enable long-term quality of track design and track geometry parameters, decrease maintenance costs, extend service life and increase competitiveness and attractiveness of railway tracks. The modernisation of railway infrastructure, which is a highly topical issue in Slovakia, is an ideal opportunity for further application of progressive systems and structural elements of railway tracks, among them also the ballastless track.

\section{Diagnostics methodology}

The experimental section diagnostics is carried out with a complex continual method by a measuring trolley $\mathrm{KRAB}^{\mathrm{TM}}$-Light. An additional method to this measuring is a partial point diagnostics with a manual track gauge. The realization and the partial results of the experimental section diagnostics are described in detail in [1].

\footnotetext{
*Corresponding author: michal.smalo@fstav.uniza.sk
} 
From the point of view of the diagnostics execution is the monitored section divided into two subsections [2]:

- section 1.1 (line No.1, the southern portal of the Tunnel Turecký vrch) and 2.1 (line No. 2, the southern portal; both sections are $175 \mathrm{~m}$ long; $102.360000-\mathrm{km} 102.535000$ ):

- $\quad \mathrm{km} 102.360000$ - km 102.460500 the ballasted track,

- $\mathrm{km} 102.460500-\mathrm{km} 102.480500$ the transition area,

- $\mathrm{km} 102.480500$ - km 102.535000 the ballastless track,

- section 1.2 (line No.1, northern portal of the Tunnel Turecký vrch) and 2.2 (line No. 2, northern portal); both sections are $640 \mathrm{~m}$ long; km 104.200000 - km 104.840 000):

- $\mathrm{km} 104.200000$ - km 104.720500 the ballastless track,

- $\mathrm{km} 104.720500-\mathrm{km} 102.740500$ the transition area,

- $\mathrm{km} 104.740500$ - km 104.840000 the ballasted track.

The measuring principle by the manual measuring trolley $\mathrm{KRAB}^{\mathrm{TM}}$-Light is contact. The measuring parameters are carried out by the touch of the scanning device with the track skeleton (rail) component of the diagnosed line. The measured results are recorded into the board computer of the measuring trolley. After the transfer to the operating computer is for each part of the experimental section created pursuant to the defined limit values of the track geometry parameters the graph of the measured track geometry parameters, table of the local faults with a list of limit values overruns and the tables of the section evaluation. The section evaluation describes the values of the standard deviations (SDO), quality marks $(Q M)$ and the quality number $(Q N)$.

The measured data are evaluated by computer in terms of provisions and according to the defined limits of the measured parameters of the track geometry. For each diagnosed subsection is created the graph of the measured parameters of the track geometry, the table of local faults with the list of the limit values overruns of the track alignment of the left and the right rail $(S L, S P)$, the track gauge $(R K)$, the change of gauge $(Z R)$, the superelevation $(P K)$, the twist fault at three different measuring plants $(Z K 1.8, Z K 6.0, Z K 12.0)$ and the vertical profile of the left and the right rail $(V L, V P)$. A part of the software evaluation is also the table of the sectional evaluation which expresses the standard deviation values, quality marks $(Q M)$ of the geometric quantities - the track alignment of the track axis $(S K)$, $R K, P K$ and the vertical profile of the rail $(V K)$ - and the quality number $(Q N)$. The evaluation respects the specifications stated in [3] and [4] for the tracks with the speed from $120 \mathrm{kmph}$ to $160 \mathrm{kmph}$ inclusive (i.e. for the speed zone - RP4). The railway infrastructure manager did not incorporate the provisions of the currently valid document [5] into the particular legislation so far. The measured values were evaluated according to the installation requirements for the acceptance of the finished works with the utilization of the new material (MSO), respectively according to the operational and safety limits for RP4 (PO1 to PO7).

The diagnostic measurements of the experimental section subsections were executed:

- the initial measurement before the construction commissioning (signed MSO) during the days 10th, 11 th July 2012, 2nd, 3rd October 2012,

- the first operational measurement (PO1) during the days 9th, 10th, 22nd April 2013,

- the second operational measurement (PO2) during the days 8th, 9th, 22nd October 2013,

- the third operational measurement (PO3) during the days 25th and 28th May 2014,

- the fourth operational measurement (PO4) on 29th October 2014,

- the fifth operational measurement (PO5) during the days 25th March 2015 and 17th April 2015,

- the sixth operational measurement (PO6) during the days 14th and 15th October 2015,

- the seventh operational measurement (PO7) during the days 22nd and 23rd March 2016,

- the eighth operational measurement (PO8) on 27th November 2016. 


\section{Diagnostics results and prognosis of the quality development}

The monitoring of the experimental section is executed from July 2012 in a half-year period. The initial measurement (MSO) was executed about 2 days before the construction commissioning. Within the initial measurement were recorded 40 local faults, 30 of which in the sections with the conventional permanent way (with the ballasted track), 9 in the section with the ballastless track and 1 local fault was diagnosed in the transition area of the section 2.1. After this measurement the contractor carried out a repair of the track geometry and a microgeometry repair of the rail heads by their grinding.

In the second operational measurement (PO2) the local faults of the vertical profile of the right $(V P)$ and the left $(V L)$ rail in the transition area of the section 1.1 were for the first time diagnosed. These faults confirm the results of the measurement by the measuring trolley of the Railways of Slovak Republic (ZSR). In the following measurements - PO3 and PO4 - was diagnosed another decrease in quality of the section 1.1, which proves the increased number of the local faults, as well as the decreasing value (increasing) of the general quality number. In November 2014 the railway infrastructure manager executed the maintenance in the transition areas of the sections 1.1 and 2.1. The diagnosed local faults were removed by means of the maintenance. The complex continual diagnostics executed by the measuring trolley $\mathrm{KRAB}^{\mathrm{TM}}$-Light and the monitoring executed by the railway infrastructure manager by means of the levelling method from December 2014 to August 2015 in the sections 1.1 and 2.1 prove other settlements of the track level. Their values do not occur as the local faults so far because the operational tolerances were not overrun. However, there is expected the formation and occurrence of the local faults within a short period of time because of the proved quality decrease tendency of the particular parameters of the mentioned sections. The sections 1.2 and 2.2 in the transition areas do not prove any faults of the values. The quality development tendency of the measurements recorded so far does not imply any probability of their occurrence either in the following measurements [68].

Within the measurements MSO, PO1, PO2 a PO3 were the quality marks of the alignment of the right (after calculating also the left) rail $\left(Q M_{S K}\right)$ classified into the interval $2<Q M \leq 3$ (recommendation: to design the repair of the track geometry into the maintenance work plan) in all of the monitored sections. The quality marks in the interval $3<Q M \leq 4$ (recommendation: to perform the repair of the track geometry till the nearest inspection) were reached in the sections 1.1 and 2.1 within the measurement PO4. After the execution of the maintenance before the measurement PO5 were the values of quality marks improved (decreased) into the interval $0<Q M \leq 2$ (the state of the track geometry is satisfactory in the evaluated section) in the section 1.1 , respectively $2<Q M \leq 3$ in the section 2.1. By the measurement PO7 was found out a slight impairment $Q M_{S K}$ in the section 1.1 to the interval $2<Q M \leq 3$, which was proved also by the measurement PO8.

The quality marks of the gauge deviation $Q M_{R K}$ are expresses in all sections and measurements in the interval $0<Q M \leq 2$, which indicates the state of the track geometry as satisfactory. The higher values of the quality marks $Q M_{R K}$ of the sections 1.1 and 2.1 result from the higher length allotment of the sections with the ballasted track construction in comparison to the sections 1.2 and 2.2 , where considerably exceeds the better quality ballastless track because of the location durability of the rails. The values $Q M_{R K}$ acquired within the monitoring executed during the operation (measurements PO1 to PO7) present the gauge as a parameter the quality of which has a permanently satisfactory development.

The quality marks of the superelevation $Q M_{P K}$ are in the intervals $0<Q M \leq 2$ in all sections and $2<Q M \leq 3$ in the measurements $\mathrm{PO} 2, \mathrm{PO} 3, \mathrm{PO} 4$ and $\mathrm{PO} 7$ in the section 1.1. The values got into the interval $0<Q M \leq 2$ after the execution of the maintenance (after the measurement PO4). The tendency of the quality marks values to increase in this section recorded within the measurement PO5 and PO6 is, however, proved as stopped by the 
measurement PO7. The quality mark $Q M_{P K}$ is placed into the interval $2<Q M \leq 3$ and in the last measurement (PO8) got the values into the interval $0<Q M \leq 2$.

As the quality mark with the worst development tendency seems to be the quality mark of the vertical profile of the rails $V P, V L Q M_{V K}$ according to the executed measurements.

The faults of the vertical elevation deviation of the rails are the most often occurring failure resulting also from the evaluation of the measurements by the measuring trolley of the ZSR. With the exception of the measuring MSO in the section 2.1 were in all measurements of the monitored sections found the values $Q M_{V K} 2<Q M \leq 3$ and in the measurements $\mathrm{PO} 1, \mathrm{PO} 2, \mathrm{PO} 3$ and $\mathrm{PO} 4$ of the section 1.1 the values $3<Q M<4$ indicating the recommendation to perform the repair of the track geometry till the nearest inspection. The values $Q M_{V K}$ decreased by the execution of the maintenance carried out in the sections 1.1 and 2.1 before the measurement PO5. The measurement PO5 and PO6 were placed into the interval $2<Q M \leq 3$. In the measurements PO7 and PO8 reached $Q M_{V K}$ the values in the interval $3<Q M<4$ in the section 2.1. The impairment tendency of the quality marks in the section 1.1 predicts the determination of the value $Q M_{V K}$ within the interval $2<Q M \leq 3$ by the measurement within a short period of time.

Within the measurements PO1, PO2, PO3 and PO4 was found out a progressive quality decrease of the sections 1.1 and 2.1 presented by the general quality number. The maintenance, executed in November 2014, by means of which the railway infrastructure manager removed the local faults preferentially of the $V K$ parameter, considerably decreased the value of the quality numbers of the particular sections from the value 2.36 (measurement PO4) to the value 1.31 (PO5) in the section 1.1 and from the value 1.86 (PO4) to the value 1.59 (PO5) in the section 2.1. The tendency of the results of the measurements PO5, PO6 and PO7 expresses the increase, which means the quality decrease of the sections 1.1 and 2.1. In the sections 1.2 and 2.2 is the quality decrease (increase $Q N$ ) only partial.

The prognosis of the quality development tendency of the particular track geometry parameters is concentrated on the problematical section from the point of view of the track quality. It concerns the transition areas in the subsections 1.1 and 2.1. From the data acquired by the measuring trolley were processed the graphs of the real deviations of the track axis alignment $(S K)$, gauge $(R K)$, gauge change $(Z R)$, superelevation $(P K)$, the projected value of which is in the mentioned section $0 \mathrm{~mm}$, the twist fault at three measuring plants $(Z K 1.8, Z K 6.0, Z K 12.0)$ and the vertical profile of the left and the right

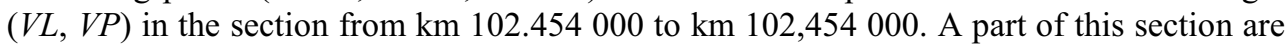
the transition areas in both track lines from $\mathrm{km} 102.460500$ to $\mathrm{km} 102.480500$.

The vertical profile of the left and the right rail $(V L, V P)$ and the development tendencies of this parameter is in regard to the maintenance planning of the transition areas the most critical parameter in the long term.

In the transition area of the section 1.1 detect the tops of the right (after calculation also the left) rail a fault of this parameter on the level of the operational limit overrun defined by the values $\pm 6 \mathrm{~mm}$ in PO4 for the right rail, respectively in PO3 and PO4 (the upper limit $\pm 6 \mathrm{~mm}$ is overrun) and in PO2, PO3 and PO4 (the lower limit $-6 \mathrm{~mm}$ ) for the left rail. The repair of the track geometry executed in the transition area in November 2014 reduced the deviation values. However, the places with the limiting values of the deviations were remained (the contact of the transition area and ballastless track beginning).

In the transition area of the section 2.1 was not recorded any overrun of the operational limits of the parameters $V L, V P$ within the PO1 to PO4. However, the railway infrastructure manager carried out the maintenance in the same time at both tracks. The maintenance executed in the transition area of the track No. 2 did not improve the deviation values of the parameter. It came to the translocation of irregularities and places with the limiting values and there was recorded an increase of the deviation $V L$ from -1.5 to $-3.5 \mathrm{~mm}$ and $V P$ from -1.0 to $-3.0 \mathrm{~mm}$ on the contact of the ballasted track with the transition area. 
The prognosis of the further quality development of the diagnosed parameters records various tendencies. The planning of the maintenance and repair works depends on the quality of the parameters which values are fastest impairing. In the case of the experimental sections in the area of portals of the Tunnel Turecký vrch it is the vertical profile of the left and the right rail in the transition areas of the subsections 1.1 and 2.1. From the tendency lines created by means of the values $V L$ and $V P$ measured within PO5, PO6, PO7 and PO8 is possible to predict that in the section 1.1 will be reached the operational limit $( \pm 6 \mathrm{~mm})$ of the parameter $V L$ probably in September 2017 and the parameter $V P$ in May 2018. In the section 2.1 will the $V L$ and $V P$ reach the value of the operational limit probably in September 2017, respectively in June 2018. The overrun levels of the operational limits should be detected within the second measurement campaign of the measuring trolley of the ZSR (in summer 2017). According to the measurement results is the railway infrastructure manager going to plan the execution of the track geometry repair in the mentioned sections.

\section{Numerical modelling of transition areas}

The results of the carried out measurements indicate that problematic parts of ballastless track sections are transitions from this structure to the ballasted superstructure. The problem of optimization of the structural solution of transition areas is a highly topical problem for the infrastructure manager, especially with regard to modernisation of corridor tracks where another ballastless track sections are planned. The decreased number of repair interventions in transition areas due to optimization of their structural solution will bring many benefits to the infrastructure manager, specifically in the form of fewer track closures and related maintenance expenses and resulting increased competitiveness of rail transport.

For the sake of optimization of transition areas, dynamic behaviour of existing transition area was analysed using 3-D models in the ANSYS software (Fig. 1 and Fig. 2). The values of vertical displacement (Fig. 3), vertical velocity (Fig. 4) and vertical acceleration (Fig. 5) of these models were then compared. In graphs, there is presented only the most interesting part - the end of ballastless track section and the beginning of transition area.

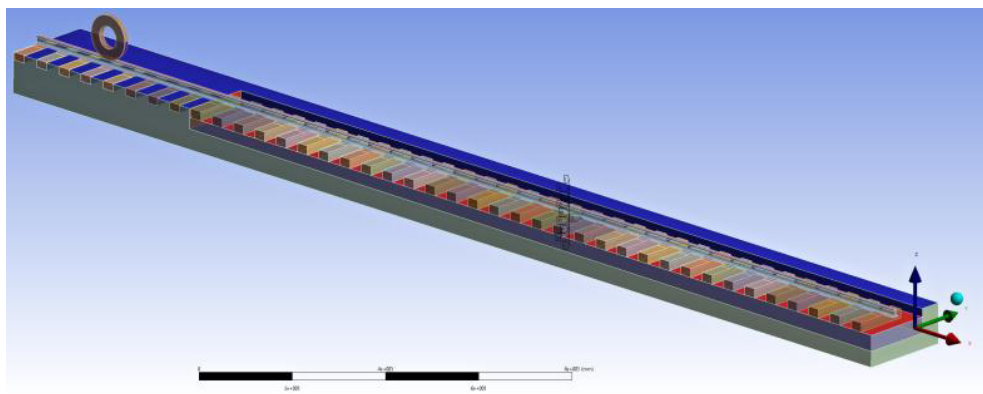

Fig. 1. Contact pairs modelling in ANSYS software.

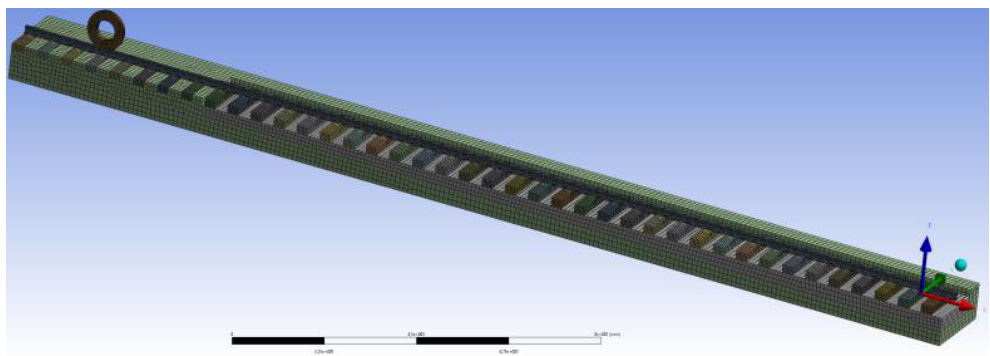

Fig. 2. Transition area model in ANSYS software. 


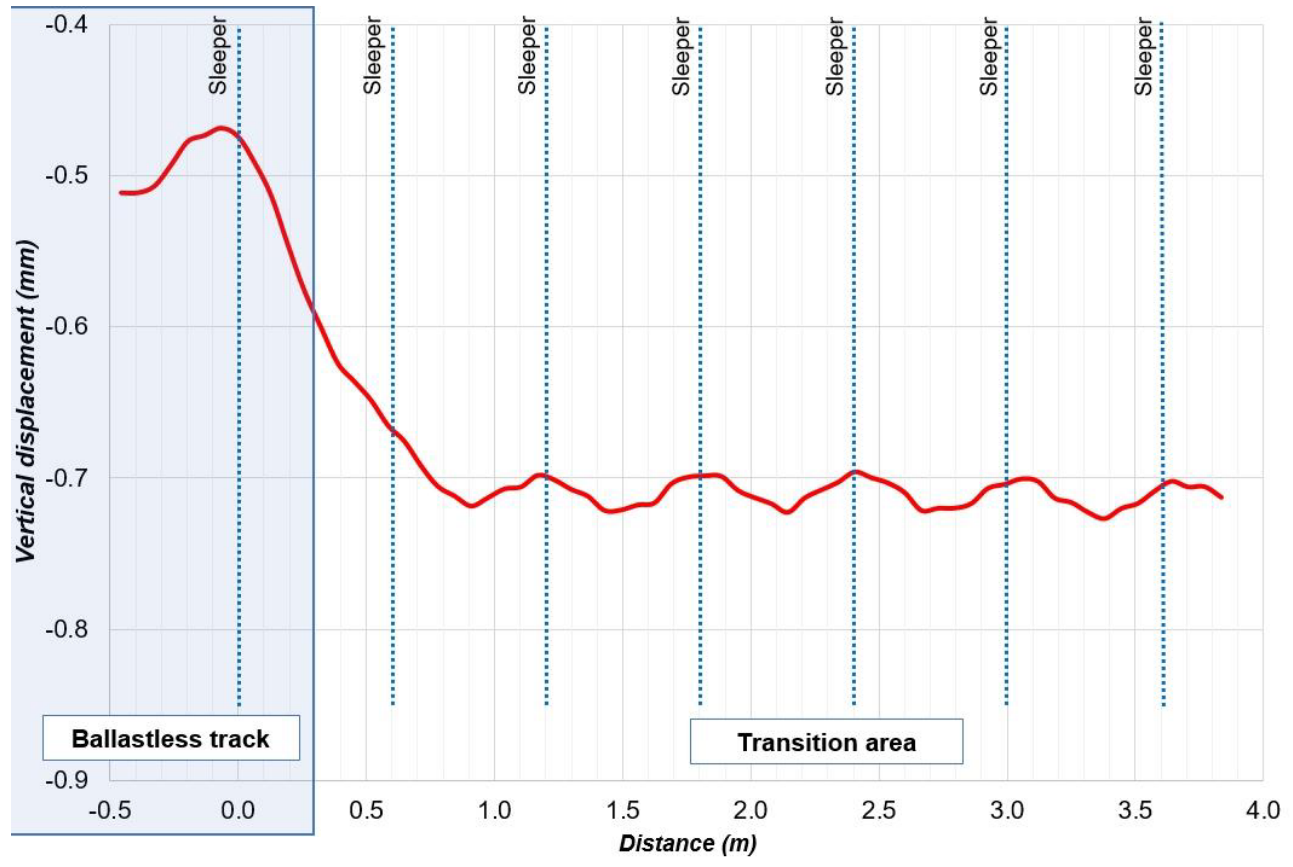

Fig. 3. Vertical displacement in transition area.

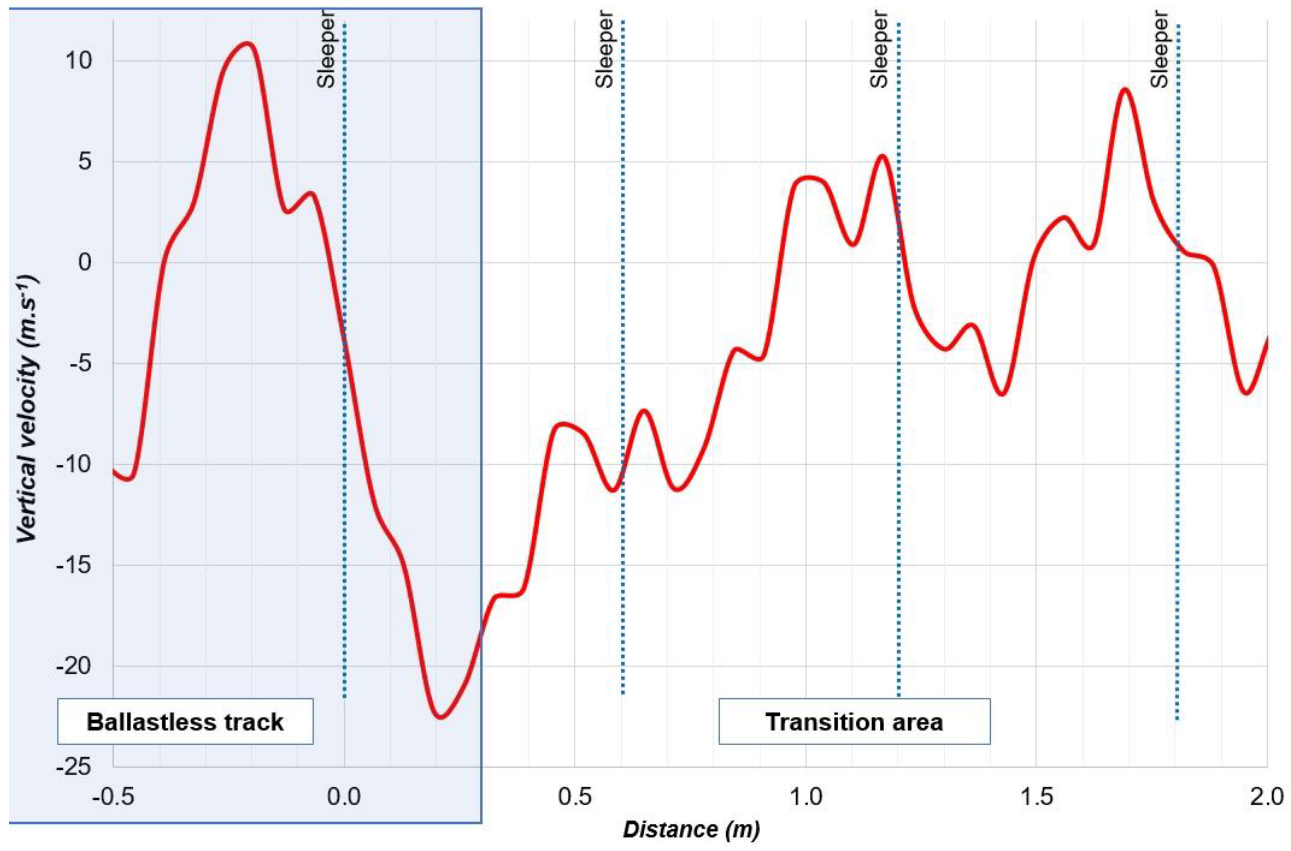

Fig. 4. Vertical velocity in transition area. 


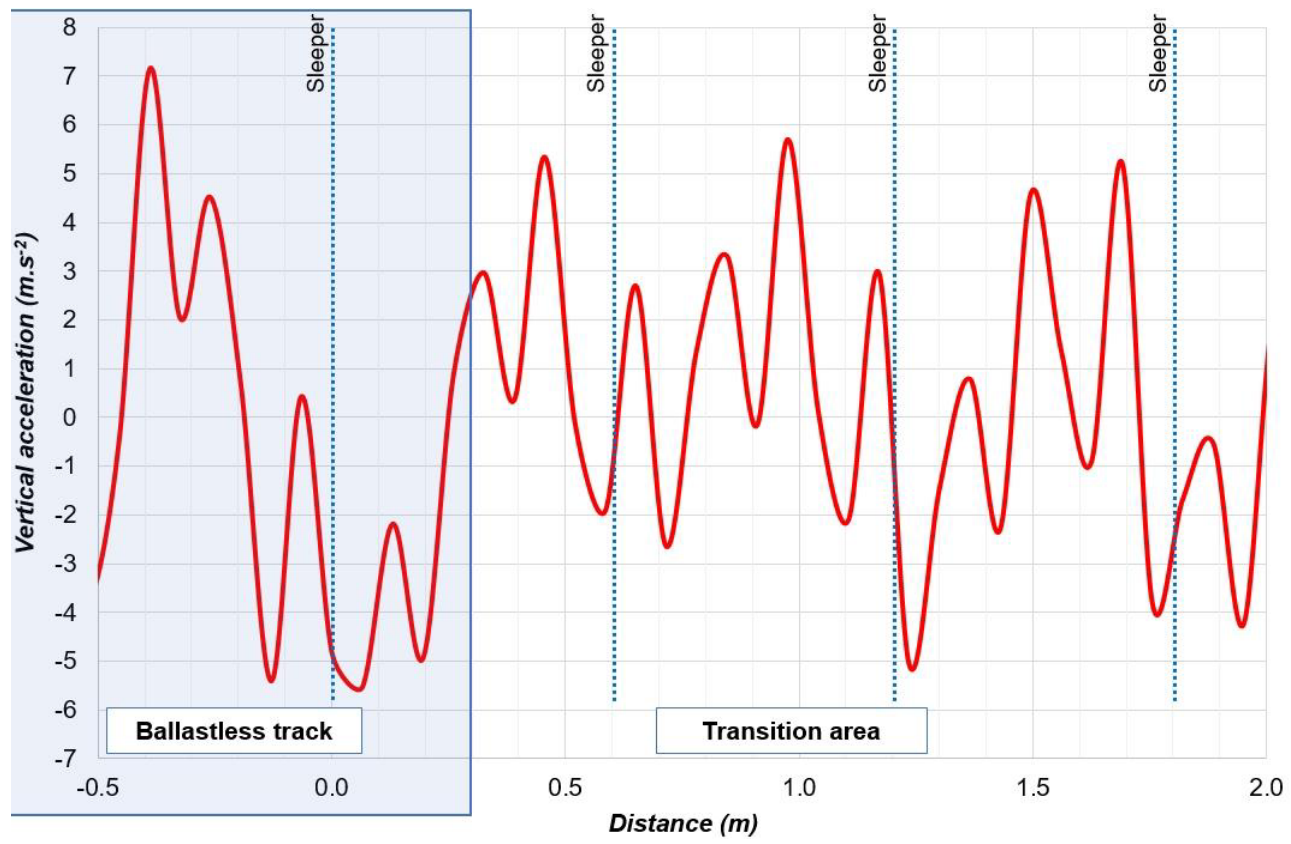

Fig. 5. Vertical acceleration in transition area.

\section{Conclusion}

The diagnostics of the track design and track geometry parameters was conducted near the portals of the Turecký vrch tunnel for five years. The measurement results were compared to the outputs of the measuring trolley of ZSR in the ballastless track sections and in the transition areas, provided by infrastructure manager. On the basis of conducted measurements and acquired results, we can conclude that the monitored ballastless sections are stable and in the period between measurements do not show any relevant changes of monitored parameters. The sections with ballasted superstructure show certain changes but they can be attributed to standard properties of this structure. The measurements of track design and track geometry by the measuring trolley $\mathrm{KRAB}^{\mathrm{TM}}$-Light indicated certain irregularities in the transition areas between the ballasted and ballastless superstructure, especially in the area of the southern tunnel portal, in both track lines. These results were subsequently confirmed by geodetic surveying and by the measuring trolley of the ZSR outputs. The lowerings recorded in these transition areas since the beginning of measurements resulted in exceeding of limit values of quality marks of top level of rails, as well as in occurrence of local errors. Due to this situation, the infrastructure manager conducted the repair intervention. This repair intervention detectably slowed down the trend of error development, but the quality of track design and track geometry of both rails is continuously degrading and further limit exceeding can be expected in the second half of 2017. This situation will certainly require another repair intervention.

The results of the carried out measurements indicate that problematic parts of ballastless track sections are transitions from this structure to the ballasted superstructure. The problem of optimization of the structural solution of transition areas is a highly topical issue for the infrastructure manager, especially with regard to modernisation of corridor tracks where another ballastless track sections are planned. The decreased number of repair interventions in transition areas due to optimization of their structural solution will bring many benefits 
to the infrastructure manager, specifically in the form of fewer track closures and related maintenance expenses and resulting increased competitiveness of rail transport.

For the sake of optimization of transition areas, dynamic behaviour transition area was monitored using 3-D model in the ANSYS software by the values of vertical displacement, vertical velocity and vertical acceleration.

In future, we are planning to compare existing transition area with optimized solution, e.g. transition area with auxiliary rails or transition area with sleepers using under-sleeper pads. On the basis of numerical modelling of these transition areas, we will try to state which of modelled transition area solution is the most suitable solution for optimization of the existing problematic transition area near the southern portal of the Turecký vrch tunnel and also for future applications of ballastless track system built within ballastless track modernisation in Slovakia.

The paper contains results of the grant VEGA 1/0275/17 Application of numerical methods to define the changes of geometrical track position.

\section{References}

1. L. Izvolt, J. Sestakova, M. Smalo, Communications - Scientific Letters of the University of Zilina, 18, 4, 19-29 (2016)

2. J. Sestakova, Z. Gocalova, Procedia Engineering, 91, 447-452 (2014), online: http://ac.els-cdn.com/S1877705814030410/1-s2.0-S1877705814030410main.pdf?_tid=7ac2c2f8-c709-11e4-aaf700000aab0f6b\&acdnat=1425980605_3c52ef455ea61af04fe1b951e4d82550

3. STN 736360 Track Alignment Design and Track Geometry of Normal-gauge Tracks (in Slovak), SUTN Bratislava (1999) and Amendment 1 (2002)

4. ZSR SR 103-7 (S) Measurement and Evaluation of Track Geometry by Measuring Trolley KRAB (in Slovak), ZSR (2008)

5. STN 73 6360-2 Railway Applications. Track. Part 2: Acceptance of Construction Works, Maintenance Works and Assessment of Service Condition Track Gauges 1435 mm (in Slovak), SUTN Bratislava (2015)

6. J. Sestakova, Civil and Environmental Engineering, 11, 1, 2-9 (2015), online: http://www.degruyter.com/dg/viewarticle.fullcontentlink:pdfeventlink/\$002fj\$002fcee. 2015.11.issue-1\$002fcee-2015-0001\$002fcee-2015-

0001.pdf?format $=I N T \& t: a c=j \$ 002$ fcee 2015 .11.issue-1 $\$ 002$ fcee-2015-0001\$002fcee2015-0001.xml

7. L. Izvolt. et. al. Monitoring of Sections of a Non-conventional Constructions of the Railway Superstructure and the Transition Areas $-7^{\text {th }}$ and $8^{\text {th }}$ Stage. ZSR Modernization of Railway Track Nove Mesto nad Vahom - Puchov, km 100.500 to 159.100, part 24-32-01 Nove Mesto - Trencianske Bohuslavice (in Slovak), University of Zilina (2015)

8. M. Smalo, L. Izvolt, Communications - Scientific Letters of the University of Zilina, 17, 4, 28-33 (2015) 